\title{
Application of Nonlinear Later TV Edition in Gigabit Ethernet
}

\author{
Hong Ma \\ Education and Training Department, Shaanxi Vocational and Technical College, Xi'an, 710100, \\ China
}

Keywords: Gigabit Ethernet. Nonlinearity. Later TV edition. Application. Code rate

\begin{abstract}
The nonlinear later TV edition is the product of computer and digital TV technology, which is com[posed of computer the corresponding nonlinear edition software on the video and audio processing cards. At present, nonlinear edition technology has a very powerful edition function, so it is applied in the TV programming. In order to obtain the higher velocity, a TV station sets up the pure gigabit Ethernet to construct the nonlinear TV edition network. According to the actual condition, the author discusses the application of nonlinear later TV edition in gigabit Ethernet, in the hope of providing some references for the relevant workers.
\end{abstract}

\section{Introduction}

With the great development of TV cause, the video code rate required is also improved continuously, to enrich the TV programs, it is required to increase the edition work sites, and ensure the stability of network, to this end, the TV station must upgrade the network and reconstruct the nonlinear later TV edition network.

\section{What is nonlinear edition}

The nonlinear edition is by contrast to the traditional linear edition dominated by the time sequence. In general, the nonlinear edition needs to be made by virtue of the digitalization and computer, and cannot separate from computer completely, because all are done in the computer. This avoids the repeated seeking on the tape and waste of time. The nonlinear edition can be arranged according to different sequences, with the advantages of convenience and rapidness. Meanwhile, it can carry out the next edition only after download for one time, and the signal quality will also not be affected. Therefore, this saves a log of equipment and labor force for the TV station. At present, most of the TV programs and films are made with nonlinear edition.

\section{Gigabit Ethernet}

Gigabit Ethernet is the past local area Ethernet, and later it was gradually developed into Ethernet, which has the advantages and characteristics of high velocity and high speed. At present, gigabit Ethernet is widely applied in many fields like finance, commerce, education and industry. In this paper, the author mainly explains the application of nonlinear edition in gigabit Ethernet.

In terms of price and compatibility, gigabit Ethernet is more advantageous than FC network, and it can reduce the restriction of low network throughput bandwidth and organize network on flexibly on this basis, making the gigabit Ethernet can carry the video and audio files with high code rate and large data volume, and can also provide the reading and writing bandwidth of real-time edition.

With the development of computer network and terminal technology, it has become a trend to adopt gigabit Ethernet to construct the nonlinear edition. In the near future, the gigabit Ethernet will gradually become the main architecture for nonlinear edition network. 


\section{Application of nonlinear later TV edition in gigabit Ethernet}

\section{Network overview}

This network adopts a HP 530 as the core server, and adopts pure gigabit network to set up the network. In the network, there are 4 edition workstations using Mtox100 nonlinear editing board cards and one workstation for dubbing. The function of the network is to complete the edition and making of a news program. When exchanging the data, the network generally adopts TCP $\backslash$ IP protocol. In addition, its network storage structure is NAS structure, which has a storage rate of $73.6 \mathrm{~GB} \times 12$ approximately to form a disk array, with HP smart array 6400 card controlling 12 scsi hard disks, and it can store approximately 100 hours' materials of AVI format, and according to the proportion of finished material, i.e. 1:4, it can edit and make 25 hours' program, so it can meet the demand for program edition of the TV station, as shown in Figure 1 for details.

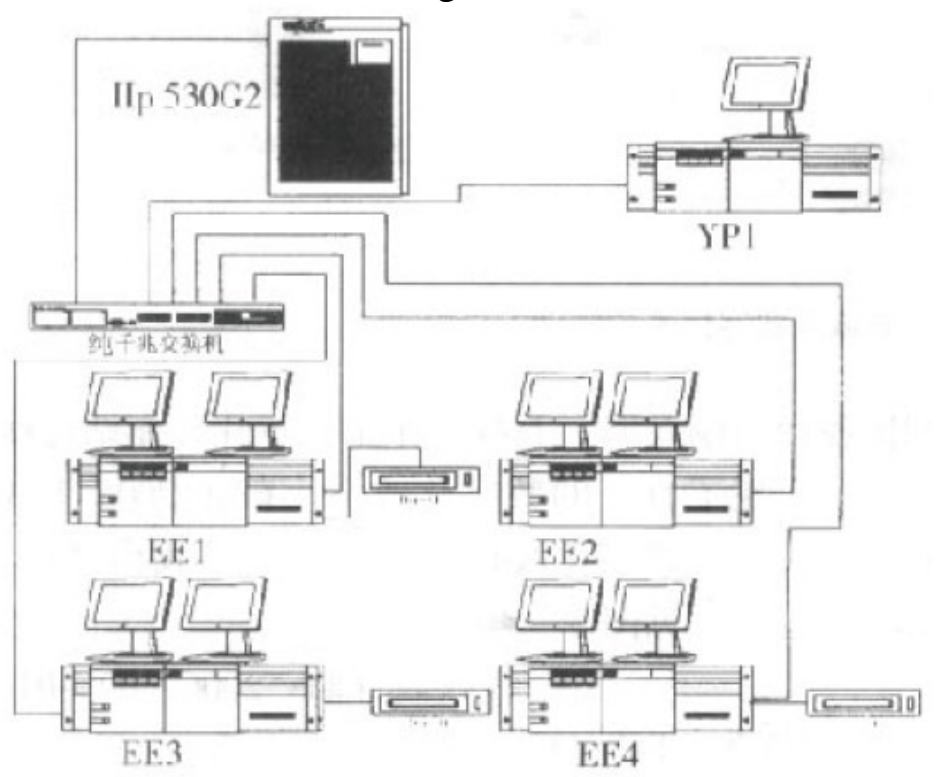

Fig. 1. News edition network connection diagram of the TV station

In the TV station, the recording format of the pre-equipment for shooting is approximately Sony DVcam format to analyze the code rate of video and audio, with video code rate of 25Mbit per second, while that of the audio $1 \mathrm{Mbit}$ per second. Calculated at the code rate, 1394 interfaces are needed to acquire the news materials. Either the speed of disk array or the network speed can meet the demand of the TV station, and the following is the specific calculation.

In the process of acquisition of news materials, the speed needed is approximately is about $4 \times 25$ $+4 \times 1+1 \times 1$, and the calculation result is 110 , therefore, the speed required is 110 Mbit per second, including 1 dubbing work station and 4 edition workstations.

The speed required for the edition process is $4 \times 3 \times 25+4+1+1 \times 1$, and the result is $305 \mathrm{Mbit}$. Therefore, the speed that must be met in the process of edition is $305 \mathrm{Mbit}$ per second, including 3-layer video work state, 1 dubbing workstation and 4 edition work stations. The following is the analysis on the array and network speed, as shown in Table 1.

Table 1. Array speed and network speed

\begin{tabular}{|l|l|l|}
\hline \multicolumn{1}{|c|}{ Name } & Network & Array \\
\hline Speed required in the process of acquisition & 110Mbit per second & 110Mbit per second \\
\hline Speed required in the process of edition & 205Mbit per second & 305Mbit per second \\
\hline Theoretical speed in the process of acquisition & 1000Mbit per second & 800Mbit per second \\
\hline Theoretical speed in the process of edition & 1000Mbit per second & 960Mbit per second \\
\hline Speed efficiency & 50\% & $60 \%$ \\
\hline Actual speed in the process of acquisition & 500Mbit per second & 480Mbit per second \\
\hline Actual speed in the process of edition & 500Mbit per second & 576Mbit per second \\
\hline
\end{tabular}




\section{\begin{tabular}{|l|l|l|}
\hline Whether the speed can meet the requirement & Yes & Yes \\
\hline
\end{tabular}}

By analyzing some problems in the actual use process, it can be concluded that the most important for this network is the speed. The speed here refers to the reading and writing speed of the disk array and the network transmission speed, and the speed is very important, which can decide whether the network can operate safely and stably. By observing the speed, it is found that this speed can fully meet the use demand of the TV station currently.

Analysis is conducted tot eh speed, including the reading and writing speed of disk array and the network transmission speed, while the reading and writing speed of the disk array is generally decided by the disk array card and the performance of physical disk. Therefore, the workers and technicians must effectively monitor the configuration of the array card and the performance of physical disk. In addition, there is also network speed, which is decided by the physical link of the speed. If the physical link of the network is not smooth, and the switchboard is not in good work state, then the transmission speed of network will also be very slow. In order to ensure the network transmission speed, it is required to monitor the network state.

\section{(II) Two nonlinear edition network architecture based on gigabit Ethernet}

\section{Nonlinear edition network architecture based on IP-SAN}

When gigabit Ethernet is used to construct the nonlinear edition network, IP-SAN is a solution with very low price. With Iscsi protocol, it combines TCOIIP with SAN technology organically, the storage disk array is directly connected to the Ethernet equipment, becoming equipment that can seek for the address in IP, and the address can be visited by its command.

iSCSI command can be embedded into the data package of TCP $\backslash$ IP, and the protocol technology of the storage equipment is connected through LAN or internet. This protocol can also merge and separate the iSCSI command and TCP\IP in the network data package transmitted.

\section{Nonlinear edition network architecture based on NAS}

NAS is a special data storage server, each application server of which uses a file management system through the network sharing protocol. A separate NAS cannot meet some requirements of nonlinear edition network, without enough storage volume, reading and writing bandwidth and system expandability, therefore, it is needed to assemble more NAS equipment through cluster technology, and this use can meet the demands.

The station of an editor must meet the bandwidth of 200Mbps, and meanwhile, it is required to guarantee that other edition sites can operate stably, and this requires to use high-end FC disk array at the rear end of NAS cluster. In this network, the architecture based on gigabit Ethernet carries many audio and video files for reading transmission and network management simultaneously. It adopts three-layer structure in network architecture, with the application server directly connected to the database, and the application sever also directly processes the client access database, in this way, the occupation to the database resource is greatly reduced and meanwhile, the data security is also guaranteed. At the same time, it is also called NAS+FC NAS method, such cluster networking method is appropriate for the construction of nonlinear edition network of very large scale. Compared with other methods, it adopts very few FC switchboard ports, without HBA, which greatly reduces the cost of TV station.

\section{Disk problems that must be noted in operation}

\section{Disk capacity}

The disk capacity must be controlled, and the use amount of the disk should be less than $80 \%$, if it exceeds $80 \%$ of the use amount, the speed of disk array will be gradually decreased, and even the network operation speed will be influenced. Therefore, the workers and technicians should clear the materials saved in the disk array irregularly, so that there is certain space and capacity in the disk for operation.

\section{Fragments of disk}

In the process of operation, the disk will generate a lot of fragments, which will influence the speed of the disk array. Therefore, when the fragments in the disk exceeds $10 \%$, the disk array speed 
will reduced sharply, if the disk fragment is up to $20 \%$, it is possible to cause halt of nonlinear edition software in use. In order to prevent this and guarantee the stability of disk, it is required to sort out and clear the fragments in the disk. The general sorting can be implemented with the sorting software in the computer, the available software includes perfect disk for servero.

\section{Disk array control card}

There is also array card in the disk, the configuration of which is very important, because it concerns the reading and writing speed of the disk array. The HP smart array 6400 array card used by the TV station is ultra $320 \mathrm{SCSI}$, with a very rapid cache speed, $128 \mathrm{M}+64 \mathrm{M}=192 \mathrm{M}$, which is very high. First, it is required to check and analyze various different parameters through the ACU application program in System Tools, and research whether these parameter are within the normal range.

In case of the configuration of HP smart array 6400, when it is used in network, the parameter configuration that can achieve the best network effect is cache read/write rate: read $25 \%$;; write $75 \%$, strip size: $128 \mathrm{~K}$.

\section{Physical disk}

Observe whether the physical disk is normal, and it is required to pay attention to the indicator lamp in operation closely. If the indicator is abnormal, it indicates that there is fault of physical disk, and it must be changed timely. If the physical disk is not changed, or the physical disk with problem is further used, the array will break down, and the data in it will be lost, which will cause very great loss.

\section{Network problems that must be noted in operation}

During operation, analysis on the network connection can enable to understand whether the current network speed meets the demands. Therefore, first it is required to observe the physical connection and network configuration.

\section{Physical connection of network}

When starting the computer, check whether the network is connected smoothly, open "My computer" to check the sharing material disk, and observe whether it is normal. If yes, the physical connection of the network is also normal.

\section{Network protocol configuration}

The above is the analysis on the physical connection, if the physical connection has been smooth, but it is still unable to open the sharing material disk, it is possible to inspect the configuration of network protocol and observe whether the network protocol is configured correctly.

\section{Inspection of hardware}

When physical connection is unavailable to the network, it is also possible to observe the hardware, the hardware to be observed include a series of equipment such as network card, switchboard and network cable. Through a series of inspections to the equipment, some problems in the network can be seen.

\section{Inspection of server and workstation}

\section{Server}

Observe the indicator lamp of the observer, if the indicator lamp of the server is normal and there is no abnormality of color change, it can be judged that its operation state is also good. In addition, it is also possible to specifically analyze the actual condition through the management tools in the operation management system, in case of error or hidden danger, timely correction and modification are required.

\section{Workstation}

The workstation is very important, the operation of which must be stable and safe, otherwise various parameters in the process of acquisition will be unreasonable and inaccurate. And the workstation also concerns whether the nonlinear edition software can operate normally and scientifically in the sharing material, and even whether the output format and routine setting in the process of transmission is closely connected to the operation of workstation. Therefore, the workers 
must have a reasonable and scientific inspection to the workstation irregularly and delete some files from the categories timely, so that the disk capacity is enough without any accident.

\section{Network safety}

As the nonlinear network of the TV station is not connected to the external network, but is only an internal LAN, it sill face the risks of external computer virus and Trojan, on the contrary, its safety threat is mainly from the internal workers. So the managers must strength management to the nonlinear edition network, establish certain measures and prevent the workers and technicians from brining the external compute virus and Trojan to the nonlinear edition network to influence the edition and making of TV programs and cause unnecessary loss.

\section{Summary and experience}

As stated above, gigabit Ethernet is a very good choice, because in the later edition of TV station, speed is very important. The gigabit Ethernet can meet the requirements of speed, and can make the network operate stably and reliably. Therefore, in order to meet the demand for the program edition of the TV station, gigabit Ethernet can be selected to construct the nonlinear edition network.

\section{References}

[1] Jiang Tao, Operation and Maintenance of Pure Gigabit Ethernet News Edition and Making Network [J]. Western Radio and Television, 2006 (10)

[2] Zhang Jin, Construction and Application of Nonlinear Edition and Making Network System of Heze TV Station [D], 2007

[3] Deng Yonghong, Nonlinear Edition Network System based on FC-Gigabit Ethernet [J], Western Radio and Television 2001(4)

[4] Niu Xuedong, Zhang Feng, A Method to Construct Nonlinear Program Making System [J] Video Technology, 2001 (2)

[5] Qian Jinghua, Design of Gigabit Ethernet Nonlinear Edition Network System

[6] Yang Yupei, Design and Perfection of Later Making Network of Wideband Video of Henan TV Station [J] Radio and Television Technology, 2000, 27 (9) 